\title{
Getting to the heart of hypopituitarism
}

\author{
Authors: Julie Martin-Grace, ${ }^{\mathrm{A}}$ Mohamed Ahmed, ${ }^{\mathrm{B}}$ Niall Mulvihill, ${ }^{\mathrm{C}}$ Eoin R Feeney ${ }^{\mathrm{D}}$ and Rachel K Crowley
}

\begin{abstract}
A 53-year-old woman was diagnosed with hypopituitarism following an acute presentation with cardiac tamponade and hyponatraemia, having recently been investigated for a pericardial effusion. Secondary hypothyroidism is a rare cause of pericardial effusion and tamponade, but an important differential to consider. Management requires appropriate hormone replacement and, critically, a low threshold for commencing stress dose steroids. Clinical signs classically associated with cardiac tamponade are frequently absent in cases of tamponade due to primary and secondary hypothyroidism, and the relatively volume deplete state of secondary hypoadrenalism in hypopituitarism may further mask an evolving tamponade, as the rise in right atrial pressure is less marked even in the presence of large effusion. Our case demonstrates the importance of a high index of suspicion for cardiac tamponade in this patient cohort, even in the absence of clinical signs, and for measuring both thyroid-stimulating hormone and thyroxine levels when evaluating a pericardial effusion.
\end{abstract}

KEYWORDS: Hypopituitarism, hypothyroidism, pericardial effusion, tamponade

\section{Case presentation}

A 53-year-old woman presented to the emergency department complaining of a short history of fever, pleuritic chest pain and exertional dyspnoea, and a history significant for secondary amenorrheoa in her twenties, previously managed as premature ovarian failure with oestrogen replacement therapy.

On examination, she was tachycardic with low-grade pyrexia. Initial investigations showed an elevated C-reactive protein and brain natriuretic peptide, but full blood count, urea and electrolytes, liver profile, cardiac enzymes and arterial blood gas were normal. Her chest X-ray showed mild cardiomegaly with clear lung fields, and her electrocardiogram showed a left bundle branch block and prolongation of the QTc interval (Fig 1). Computerised tomography pulmonary angiography

\footnotetext{
Authors: A senior house officer, St. Vincent's University Hospital, Dublin, Ireland; 'Bregistrar, St. Vincent's University Hospital, Dublin, Ireland; ' Consultant cardiologist, St. Vincent's University Hospital, Dublin, Ireland; ${ }^{D}$ consultant infectious disease physician, St. Vincent's University Hospital and University College Dublin, Dublin, Ireland; ${ }^{E}$ consultant endocrinologist, St. Vincent's University Hospital and University College Dublin, Dublin, Ireland
}

was performed to exclude a pulmonary embolus. This showed a large pericardial effusion with reflux of contrast into the right atrium, consistent with a functional tricuspid regurgitation. Echocardiogram confirmed a global simple pericardial effusion with no evidence of cardiac tamponade or haemodynamic compromise. The treating team were advised to manage supportively and investigate for the cause of the effusion.

\section{Differential diagnosis}

The differential diagnosis for a pericardial effusion includes idiopathic, viral pericarditis, uraemia, heart failure and postcardiac intervention. Less common causes include tuberculosis (TB), fungal or bacterial pericarditis, malignancy, systemic lupus erythematosus, post-myocardial infarction (Dressler's syndrome), radiation and hypothyroidism. ${ }^{1}$ Normal troponins, renal profile and autoimmune screen focused the investigations on an infectious cause. Thyroid-stimulating hormone (TSH) was measured at this point, which was within normal limits, but free thyroxine (T4) was not. Blood cultures, viral serology and quantiferon testing were negative, and nothing in her history, examination or imaging suggested an underlying neoplastic process. Her symptoms improved and C-reactive protein normalised with supportive care alone. She was discharged with a diagnosis of effusion secondary to viral pericarditis.

\section{Case progression and outcome}

The patient presented 2 weeks later with acutely worsened exertional dyspnoea, markedly increased cardiomegaly on chest X-ray (Fig 2A), and new hypovolaemic hyponatraemia $\left(\mathrm{Na}^{+} 123 \mathrm{mmol} / \mathrm{L}\right.$, serum osmolality $\left.248 \mathrm{mOsm} / \mathrm{kg}\right)$. Despite no clinical evidence of tamponade, echocardiogram (Fig 2B showed diastolic right atrial collapse consistent with cardiac tamponade. A pericardial drain was inserted, which yielded $1,150 \mathrm{~mL}$ of fluid consistent with an exudative effusion. Subsequent culture, gram stain, viral polymerase chain reaction, TB culture and cytological examination of the fluid were negative.

A random cortisol was sent in because of the development of acute hyponatraemia and was inappropriately low $(43 \mathrm{nmol} / \mathrm{L})$. High-dose intravenous hydrocortisone was commenced and an endocrinology opinion was requested; they noted her cool, pale skin and history of secondary amenorrhoea. Pituitary function investigations (Table 1) were consistent with hypopituitarism and magnetic resonance imaging pituitary (Fig 2C and 2D) showed an empty sella. Her pericardial drain was removed and repeat echocardiogram excluded reaccumulation of the 


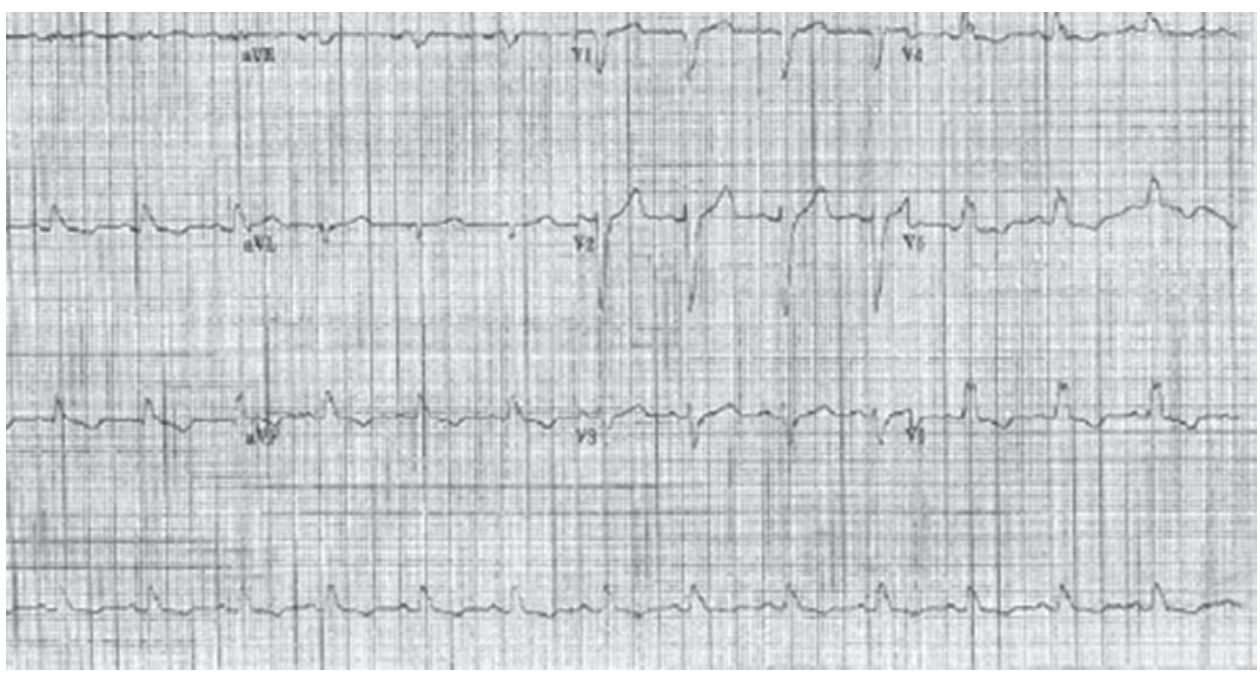

Fig 1. Electrocardiogram demonstrating a left bundle branch black and prolongation of the QTc interval. pericardial effusion. Her sodium improved to $134 \mathrm{mmol} / \mathrm{L}$ following 3 days of steroid replacement and fluid rehydration. She was discharged on oral hydrocortisone and levothyroxine, and referred to endocrinology services for ongoing management of hypopituitarism and further assessment of her growth hormone status.

\section{Discussion}

Pericardial effusion in hypothyroidism and hypopituitarism

Pericardial effusion occurs in 3-6\% of all cases of hypothyroidism, which resolve on regaining euthyroid status.
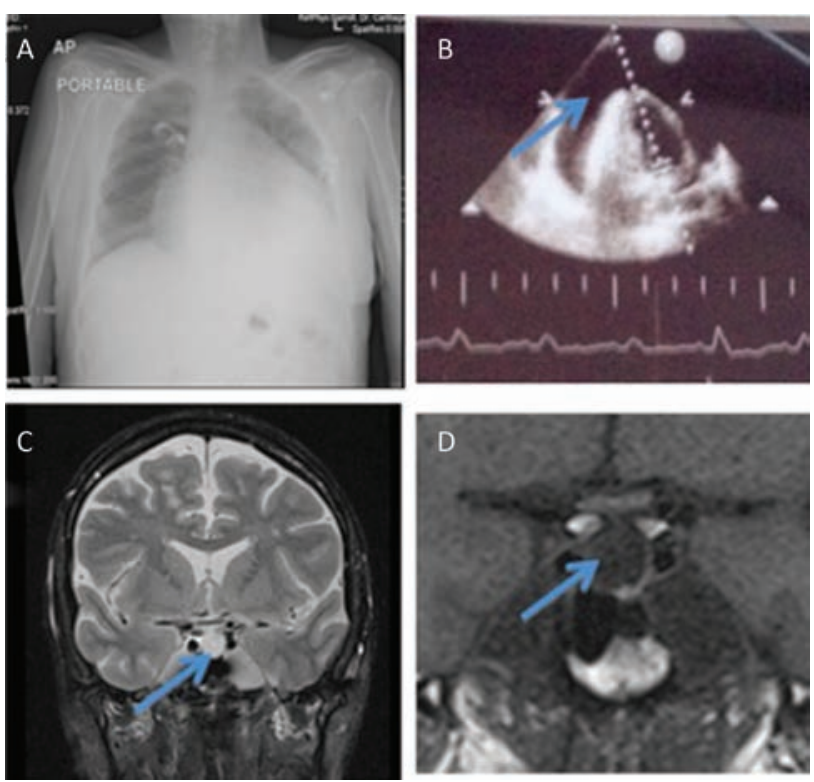

Fig 2. A - portable chest X-ray demonstrating cardiomegaly; B echocardiogram showing $2 \mathrm{~cm}$ simple global pericardial effusion; C T2-weighted MRI pituitary; D - T1-weighted MRI pituitary. Arrows mark empty sella.
These effusions rarely progress to cause tamponade, as the accumulation of fluid is slow, allowing the pericardium to distend to accommodate it. The majority of patients are diagnosed with hypothyroidism and commenced on levothyroxine replacement before a haemodynamically significant effusion can develop. ${ }^{2}$ This case is one of only a handful of reported cases of tamponade due to secondary hypothyroidism in previously undiagnosed hypopituitarism

Clinical signs of cardiac tamponade, such as tachycardia, pulsus paradoxus and jugular venous distension (which in association with muffled heart sounds and hypotension make up Beck's Triad), ${ }^{3}$ are frequently absent in both primary and secondary hypothyroid patients. A small study of tamponade associated with primary hypothyroidism noted lower heart rates in the hypothyroid tamponade cases $(80.75 \pm 13$ beats/min)

\begin{tabular}{lll}
\multicolumn{3}{l}{ Table 1. Anterior pituitary hormones } \\
\hline Investigation & Result & Reference range \\
Growth hormone & $0.03 \mu \mathrm{g} / \mathrm{L}$ & $0.13-9.9 \mu \mathrm{g} / \mathrm{L}$ \\
Cortisol (random) & $48 \mathrm{nmol} / \mathrm{L}$ & $\mathrm{AM} 171-536 \mathrm{nmol} / \mathrm{L}$ \\
$\mathrm{PM} \mathrm{64-327} \mathrm{nmol/L}$ & & \\
$\mathrm{LH}$ & $<0.5 \mathrm{IU} / \mathrm{L}$ & $8-59 \mathrm{U} / \mathrm{L}$ (menopausal) \\
FSH & $<0.5 \mathrm{IU} / \mathrm{L}$ & $26-135 \mathrm{U} / \mathrm{L}$ (menopausal) \\
TSH & $0.73 \mathrm{mIu} / \mathrm{L}$ & $0.27-4.2 \mathrm{mI} / \mathrm{L}$ \\
FT4 & $6.3 \mathrm{pmol} / \mathrm{L}$ & $12.0-22.0 \mathrm{pmol} / \mathrm{L}$ \\
T3 & $0.44 \mathrm{nmol} / \mathrm{L}$ & $1.3-3.1 \mathrm{nmol} / \mathrm{L}$ \\
Total prolactin & $184 \mathrm{mU} / \mathrm{L}$ & $102-496 \mathrm{mU} / \mathrm{L}$ \\
\hline
\end{tabular}

$\mathrm{FSH}=$ follicle stimulating hormone; FT4 = free thyroxine; $\mathrm{LH}=$ luteinising hormone; TSH = Thyroid-stimulating hormone.

Investigation of pituitary function showing cortisol insufficiency, undetectable gonadotropins in a postmenopausal female, secondary hypothyroidism and normal prolactin, consistent with hypopituitarism. Previous oestradiol, FSH/ LH levels sent from primary care reviewed retrospectively were consistent with hypogonadotrophic hypogonadism, suggesting pituitary dysfunction preceded this presentation by a number of years. At the time of writing, growth hormone levels were not detectable but the patient had not yet undergone dynamic pituitary function testing to confirm growth hormone deficiency. 
compared with euthyroid cases $(112 \pm 12.8$ beats/min, $\mathrm{p}<0.01)$, and suggested that hypothyroidism be considered in the differential of a patient with a significant pericardial effusion without a tachycardic response. ${ }^{4}$

This pattern is not observed in primary adrenal insufficiency, suggesting that it is the secondary hypothyroidism rather than cortisol deficiency that causes an effusion to develop in hypopituitarism. However, cortisol deficiency in hypopituitarism may mask progression from pericardial effusion to tamponade, by contributing to a relatively volume deplete state; therefore, the rise in right atrial pressure as an effusion accumulates is less marked. This may explain the absence of an elevated jugular venous pressure. ${ }^{5}$

\section{Investigation of a pericardial effusion}

First line investigations include electrocardiogram, chest $\mathrm{X}$-ray, inflammatory markers, full blood count, renal profile and echocardiogram to assess the haemodynamic impact of the effusion, and look for the common causes. Further investigations include $\mathrm{TSH},{ }^{1}$ rheumatoid factor, erythrocyte sedimentation rate, anti-nuclear antibodies, blood cultures and further imaging as appropriate. If available, pericardial fluid samples should be analysed for protein and lactate dehydrogenase content, and culture, gram staining, virology, TB culture and cytological examination should be performed. ${ }^{1}$ In cases where the aetiology remains unclear with no evidence of haemodynamic compromise, a trial of empiric antiinflammatory therapy is reasonable. ${ }^{3}$

TSH measurement alone will detect the majority of cases of hypothyroidism, but will be misleading in the setting of central hypothyroidism. While central hypothyroidism is a very rare cause of tamponade, our case demonstrates the importance of measuring both a serum TSH and free T4 to ensure that cases of central hypothyroidism do not go undetected, not only to facilitate directed therapy to prevent reaccumulation, but also to prompt investigation and appropriate replacement of other anterior pituitary hormones, most notably cortisol.

\section{Key learning points}

$>$ Cardiac tamponade is a rare but reported presentation of hypothyroidism and hypopituitarism, diagnosis of which is important to prevent progression or re-accumulation of a pericardial effusion.
> There are features common to cardiac tamponade due to both primary hypothyroidism and hypopituitarism, including the absence of classical signs such as tachycardia, raised jugular venous pressue and pulsus paradoxus. Therefore, it is important to maintain a high index of suspicion for cardiac tamponade in this subset of patients with large pericardial effusions, even in the absence of clinical signs.

> Both thyroid stimulating hormone and free T4/triiodothyronine should be measured when investigating a pericardial effusion to avoid missing secondary hypothyroidism.

\section{Conflicts of interest}

The authors have no conflicts of interest to declare.

\section{Acknowledgments}

Written consent has been obtained from the patient to publish the clinical details and images in this article.

\section{References}

1 Assessment of pericardial effusion. London: BMJ Best Practice, 2015. http://bestpractice.bmj.com/best-practice/monograph/458.html [Accessed 15 February 2017].

2 Guberman BA, Fowler NO, Engel PJ et al. Cardiac tamponade in medical patients. Circulation 1981;64:633-40.

3 Alder Y, Charron P, Imazio M et al. 2015 ESC Guidelines for the diagnosis and management of pericardial diseases: The Task Force for the Diagnosis and Management of Pericardial Diseases of the European Society of Cardiology (ESC)Endorsed by: The European Association for Cardio-Thoracic Surgery (EACTS). Eur Heart $J$ 2015;36:2921-64.

4 Wang JL, Hsieh MJ, Lee CH. Hypothyroid cardiac tamponade: clinical features, electrocardiography, pericardial fluid and management. Am J Med Sci 2010;340:276-81.

5 Goswami R, Tandon M, Singh B, Shah P, Ammini AC. Circulatory collapse in a 30-year-old amenorrheic woman. Postgrad Med J 1996:72:501-4.

Address for correspondence: $\mathrm{Dr}$ ] Martin-Grace, Department of Endocrinology \& Diabetes Mellitus, St. Vincent's University Hospital, Elm Park, Dublin 4, Ireland.

Email: Julie.martingrace@gmail.com 